\title{
Formulation of long-wavelength indocyanine green nanocarriers
}

\author{
Vikram J. Pansare \\ William J. Faenza \\ Hoang $\mathrm{Lu}$ \\ Douglas H. Adamson \\ Robert K. Prud'homme
}




\title{
Formulation of long-wavelength indocyanine green nanocarriers
}

\author{
Vikram J. Pansare, ${ }^{a}$ William J. Faenza, ${ }^{b}$ Hoang Lu, ${ }^{a}$ Douglas H. Adamson, ${ }^{c}$ and Robert K. Prud'homme ${ }^{a, \star}$ \\ aprinceton University, Department of Chemical and Biological Engineering, Princeton, New Jersey, United States \\ ${ }^{\mathrm{b}}$ Persis Science LLC, Princeton, New Jersey, United States \\ 'University of Connecticut, Department of Chemistry, Storrs, Connecticut, United States
}

\begin{abstract}
Indocyanine green (ICG), a Food and Drug Administration (FDA)-approved fluorophore with excitation and emission wavelengths inside the "optical imaging window," has been incorporated into nanocarriers (NCs) to achieve enhanced circulation time, targeting, and real-time tracking in vivo. While previous studies transferred ICG exogenously into NCs, here, a one-step rapid precipitation process [flash nanoprecipitation (FNP)] creates ICG-loaded NCs with tunable, narrow size distributions from 30 to $180 \mathrm{~nm}$. A hydrophobic ion pair of ICGtetraoctylammonium or tetradodecylammonium chloride is formed either in situ during FNP or preformed then introduced into the FNP feed stream. The NCs are formulated with cores comprising either vitamin E (VE) or polystyrene (PS). ICG core loadings of $30 \mathrm{wt}$. \% for VE and $10 \mathrm{wt}$. \% for PS are achieved. However, due to a combination of molecular aggregation and Förster quenching, maximum fluorescence (FL) occurs at $10 \mathrm{wt}$ \% core loading. The FL-per-particle scales with core diameter to the third power, showing that FNP enables uniform volume encapsulation. By varying the ICG counter-ion ratio, encapsulation efficiencies above $80 \%$ are achieved even in the absence of ion pairing, which rises to $100 \%$ with $1: 1$ ion pairing. Finally, while ICG ion pairs are shown to be stable in buffer, they partition out of $\mathrm{NC}$ cores in under $30 \mathrm{~min}$ in the presence of physiological albumin concentrations. ๑ 2017 Society of Photo-Optical Instrumentation Engineers (SPIE) [DOI: 10.1117/1.JBO.22.9.096007]
\end{abstract}

Keywords: long-wavelength imaging; biomedical imaging; indocyanine green; nanocarriers; nanoparticles; stability.

Paper 160231RRR received May 22, 2016; accepted for publication Jul. 26, 2017; published online Sep. 18, 2017.

\section{Introduction}

The field of optical/near-infrared (NIR) imaging has gained importance in recent years as an alternative to radioactive imaging techniques for in vivo diagnostics. Contrast agents with excitation and/or emission wavelengths in the range from 700 to $1450 \mathrm{~nm}$ fall into the "optical imaging window," a region where absorbance and autofluorescence from blood and tissue components are minimized. ${ }^{1}$

Nanocarriers (NCs) are of interest as therapeutic delivery agents, ${ }^{2-5}$ as targeted imaging agents for diagnosis, ${ }^{1,6-9}$ and, in the dual role of therapy and diagnosis, as "theranostic" agents. ${ }^{10-15}$ Most often, NCs for therapeutic applications are rendered fluorescent by postfunctionalizing the surface of the NC with a fluorescent dye. ${ }^{16-23}$ The main concerns with this approach are that (1) far fewer agents can be incorporated on the surface versus in the interior before the onset of quenching and (2) the dyes may interfere with targeting ligands placed on the NC surface (ligands that are often present in relatively low amounts).

The long-wavelength imaging agent indocyanine green [ICG, also known as Cardiogreen, see Fig. 1(a)], a Food and Drug Administration (FDA)-approved medical imaging dye, has been implemented by the medical community to assess hepatic and cardiological function in humans. ${ }^{24}$ ICG is attractive as an imaging agent due to its $772 / 831 \mathrm{~nm}$ absorption/emission wavelengths [Fig. 1(d)], which fall into the optical imaging window (700 to $1450 \mathrm{~nm}$ ), its long history of use in humans,

*Address all correspondence to: Robert K. Prud'homme, E-mail: prudhomm@ princeton.edu and its approval by the FDA. Therefore, there is considerable interest in incorporating ICG into nanoscale carriers such as liposomes, ${ }^{25,26}$ micelles, ${ }^{15,27-30}$ nanocapsules, ${ }^{31,32}$ and mesocapsules, ${ }^{33}$ as well as in more complex formulations for dual purpose diagnostics and therapy, ${ }^{11,34}$ multimodal imaging ${ }^{35}$ systems that can enhance the apparent quantum yield (QY) of ICG to several times its natural QY in water, ${ }^{34}$ ICG embedded within hydrophobic polymers, ${ }^{36,37}$ and mesoporous materials. ${ }^{38}$ ICG has been incorporated into these carriers either as the pure molecule, ${ }^{28}$ as a complex with a hydrophobic cationic species, ${ }^{29}$ or as a conjugate to polymers. ${ }^{30}$ Ionic complexes of ICG are particularly attractive as they do not require new approval by the FDA for biomedical use and have a much shorter path to clinical adoption.

Although many types of delivery systems have been studied for the delivery of ICG, several with in vivo data for both targeted and nontargeted formulations, there is a notable lack of data on the stability of the delivery systems under physiological conditions. The delivery constructs (such as liposomes or micelles) might be stable, but the ICG may not be stably incorporated into the constructs. ICG's unique amphiphilic structure, with both hydrophobic and ionic domains, enables incorporation into particles but also drives strong binding to proteins such as albumin. Therefore, exchange between the particle and serum proteins can be a significant issue for long-term imaging studies. Physical instability is related to imaging stability, since ICG bound to proteins can exhibit significantly higher fluorescence (FL) QYs than in particles, ${ }^{39}$ and, therefore, in vivo

1083-3668/2017/\$25.00 @ 2017 SPIE 
(a)

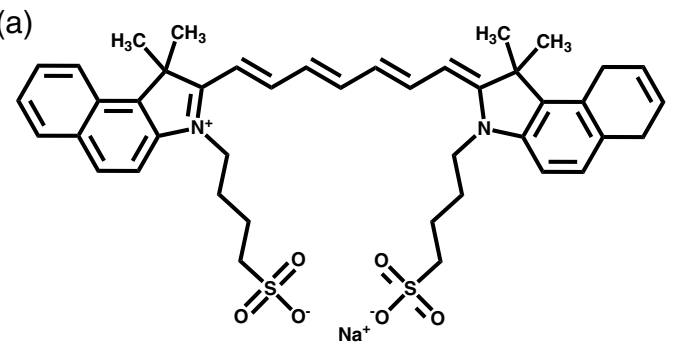

(b)

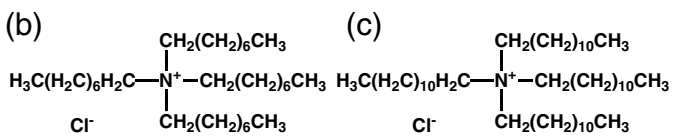

(e)

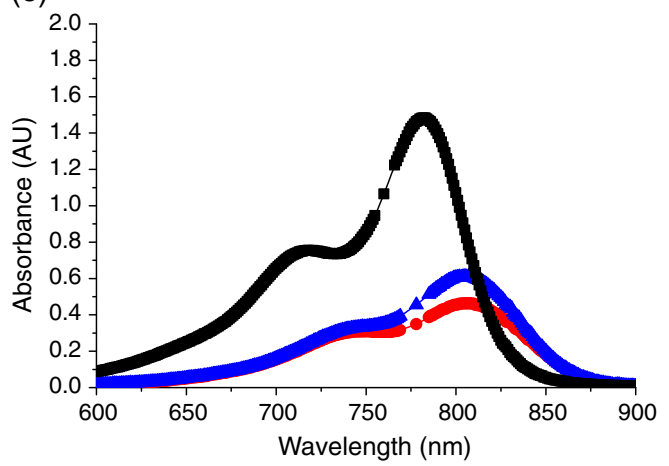

(d)

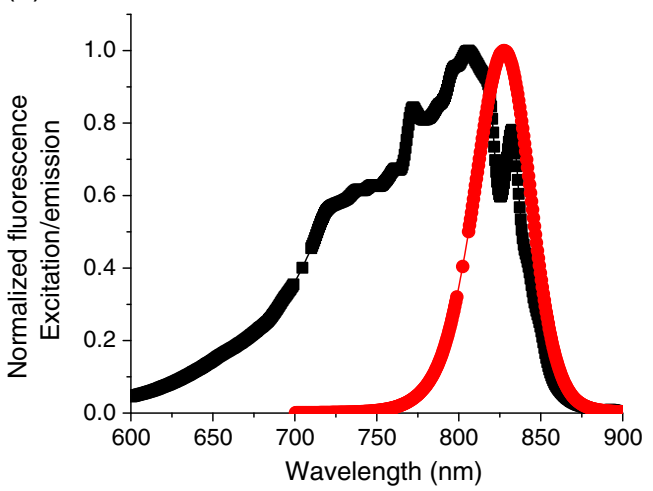

(f)

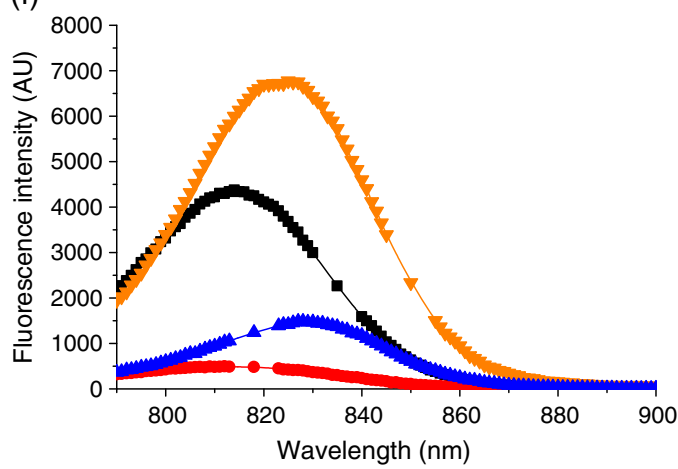

Fig. 1 (a) Structure of ICG, (b) structure of TOAC, (c) structure of TDDAC, (d) normalized FL excitation and emission spectra for ICG in DMSO (ø excitation, • emission). Excitation wavelength used was $772 \mathrm{~nm}$, whereas the emission peak varied between 810 and $831 \mathrm{~nm}$ depending on whether the ICG was free in solution or NC-encapsulated. (e) and (f) Absorbance and FL spectra of ICG formulations at $[\mathrm{ICG}]=0.073 \mathrm{mg} / \mathrm{mL}$ (- free ICG in water, $\boldsymbol{\nabla}$ ICG +4 wt. \% BSA in phosphate buffer, $\bullet$ ICG-C ${ }_{8}$ in situ complex at $10 \mathrm{wt}$. \% loading in PS-b-PEG micelles, $\triangle$ ICG-C $\mathrm{C}_{8}$ in situ complex at $10 \mathrm{wt}$ \% loading in VE + PS- $b$-PEG NCs). Absorbance curves in (e) and lower in magnitude and red-shifted relative to the free ICG curve indicating the formation of aggregates within the NC core. FL curves in (f) show the brightness of ICG increases almost twofold upon incubation with $40 \mathrm{mg} / \mathrm{mL}$ BSA (comparable to physiological protein levels) but decreases significantly upon complexation and encapsulation within block copolymer micelles or NCs with VE cores. Gaps in data to show symbols clearly.

studies intended to track the biodistribution of NCs over time may be confounded by the signal from ICG-protein complexes.

Our objectives are fourfold: (1) to develop a robust assembly process to prepare ICG-loaded NCs over a range of sizes, (2) to demonstrate that the ICG is uniformly distributed within the NC core, (3) to use ion pairing to incorporate ICG in the NC core, and (4) to assess the stability of ICG NCs under physiologically relevant conditions.

\section{Results and Discussion}

\subsection{ICG Structure and Optical Properties}

The structures of ICG and the tetraoctylammonium chloride (TOAC) and tetradodecylammonium chloride (TDDAC) molecules are shown in Figs. 1(a)-1(c). ICG possesses an amphiphilic structure with aromatic groups, a conjugated backbone, and charged sulfonate groups. Its overall charge is -1 . Thus, it complexes with the cationic ammonium salts TOAC and TDDAC, which are known as "phase transfer catalysts" with strong interfacial activity. ${ }^{40}$ We use these compounds instead of the more commonly used butyl variant $\left(\mathrm{C}_{4}\right)$ due to their greater hydrophobicity, which we anticipate would increase NC stability against ICG ion exchange. ICG has a broad excitation spectrum [Fig. 1(d)] with emission at 810 to $830 \mathrm{~nm}$ depending on the ICG concentration, solvent, and NC encapsulation. An excitation wavelength of $772 \mathrm{~nm}$ was used for all experiments. These wavelengths fall into the optical imaging window where maximum optical penetration is possible.

The basic optical properties of free ICG in solution and encapsulated in NC form are shown in Table 1 and Figs. 1(e) and 1(f). The generally accepted QY for free ICG is $1.3 \%{ }^{11,24}$ Relative FL measurements with one of our ICG-containing NC systems approximate the apparent QY for the NCs as $0.44 \%$. Figure 1 (e) shows that ICG encapsulated with NC systems exhibits lower absorbance values and red-shifting relative to free ICG. This, combined with the lower FL values and redshifting in the FL spectrum in Fig. 1(f), indicates that the formation of ICG molecular aggregates in the NC core. Aggregate formation is a key mechanism of FL quenching. ${ }^{41,42}$

\subsection{NC Formation and Maximizing ICG NC Intrinsic Fluorescence}

The key platform underlying NC fabrication is flash nanoprecipitation (FNP), as described by Johnson and Prud'homme. ${ }^{43}$ The process enables coencapsulation of a variety of drugs, $4,5,44$ pesticides, ${ }^{45}$ organic imaging agents, ${ }^{9,46}$ nanocrystals,,${ }^{47}$ and 
Table 1 Optical properties of free ICG and ICG in NCs.

\begin{tabular}{|c|c|c|}
\hline Optical property & $\begin{array}{l}\text { Free ICG } \\
\text { in water }\end{array}$ & $\begin{array}{l}\text { ICG NC } \\
\text { in water }\end{array}$ \\
\hline QY (\%) & $1.3^{11}$ & $0.44^{\mathrm{b}}$ \\
\hline $\begin{array}{l}\text { Absorbance max wavelength }(\mathrm{nm}) \\
\text { and extinction coefficient }\left(\mathrm{M}^{-1} \mathrm{~cm}^{-1}\right)\end{array}$ & $\begin{array}{l}783 \text { and } \\
1.6 \times 10^{5}\end{array}$ & $\begin{array}{l}804 \text { and } \\
6.3 \times 10^{4}\end{array}$ \\
\hline Intrinsic FL (slope) $[\mathrm{AU} /(\mathrm{mg} / \mathrm{mL})]$ & $1.8 \times 10^{7}$ & $6.6 \times 10^{5}$ \\
\hline $\begin{array}{l}\text { FL excitation and emission } \\
\text { wavelengths }(\mathrm{nm})\end{array}$ & $772 / 810$ & $\begin{array}{l}772 / 810 \\
\text { to } 830^{c}\end{array}$ \\
\hline
\end{tabular}

a Formulation: ICG- $\mathrm{C}_{8}$ in situ, 10 wt. \% ICG core loading.

${ }^{\mathrm{b}}$ Determined by relative FL against free ICG.

cBroad excitation spectrum with peak at $772 \mathrm{~nm}$ used for all experiments.

metal clusters. In the process, hydrophobic species and an amphiphilic block copolymer in a water-miscible organic stream [often tetrahydrofuran (THF)] are rapidly mixed against an antisolvent stream (usually water). The key to the process is the specially designed micromixing cavities that create supersaturations as high as 10,000 in $1.5 \mathrm{~ms} .{ }^{5} \mathrm{NCs}$ were formed by making hydrophobic ICG:cationic counter-ion complexes in two ways to form ICG NCs: one consists of preforming the hydrophobic ICG-ammonium salt complex ("premade complex") then forming NCs and the second consists of forming the ICGcationic salt hydrophobic complex in situ during nanoprecipitation ("in situ"). The process conditions for each formulation are presented in Sec. 4. Zeta potentials were measured for the NC systems created (shown in Table 2) and were found to range between -1 and $0 \mathrm{mV}$ as expected due to the charge screening effects of the PEG shell around each $\mathrm{NC}^{48}$

A hydrophobic excipient such as vitamin E (VE) or polystyrene (PS) is often used in the NC formulation to improve the capture and stability of the active ingredient, ICG complex in this case, and to define the $\mathrm{NC}$ size. In addition, as explained previously, ${ }^{46}$ the hydrophobic excipients uniformly distribute the dyes within NC cores and reduce quenching. Although increasing the number of dye molecules per NC initially increases the FL per NC, ICG molecular aggregation ${ }^{24,49}$ and the rate of Förster resonant energy transfer ${ }^{46,50,51}$ increase, causing FL quenching, which results in a decrease in FL per NC (intrinsic FL). Thus, there is an optimum in the NC dye core loading to achieve the maximum FL per NC. As seen in Fig. 2, the ICG complex core loading, which gives the maximum FL for the in situ formed ICG-TOAC (ICG-C 8 ) system, is $10 \mathrm{wt}$. $\%$. The calculation procedure for estimating the number concentration of NCs is given in Ref. 46. The core loading of the ICG complex is defined as

Table 2 Zeta potential of ICG NCs.

\begin{tabular}{lc} 
Sample & Zeta potential $(\mathrm{mV})$ \\
\hline $\mathrm{ICG}_{\mathrm{C}}$ in PS- $b$-PEG micelles & -0.22 \\
$\mathrm{ICG}-\mathrm{C}_{8}$ in VE + PS- $b$-PEG NCs & -0.88 \\
\hline
\end{tabular}

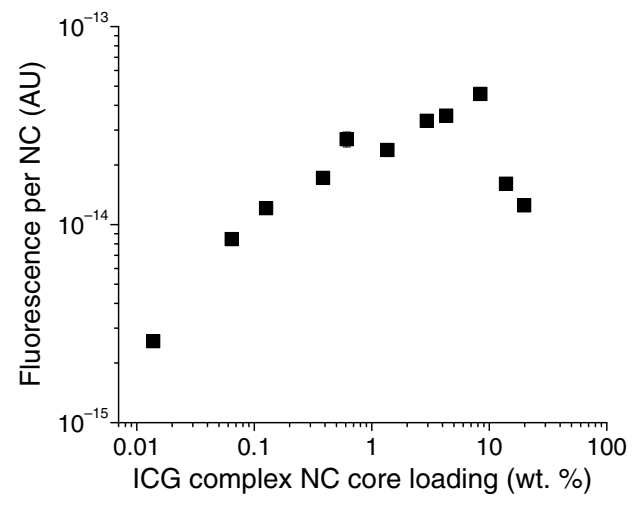

Fig. 2 FL per NC versus ICG complex core loading. The maximum in FL per NC (highest intrinsic FL) for $100 \mathrm{~nm} \mathrm{NCs} \mathrm{was} \mathrm{found} \mathrm{at}$ $\sim 10$ wt.\% ICG complex core loading. Subsequent ICG- $\mathrm{C}_{8}$ complex NCs were formulated with this optimal loading. A measure of the error is provided at the data point at 0.61 wt. \%: $2.54 \times 10^{-14}$ $\pm 2.33 \times 10^{-15} \mathrm{AU}$.

ICG complex core loading

$$
=\frac{[\text { ICG complex }]}{[\text { ICG complex }]+[\text { excipient }]+[\text { PS-block }]},
$$

where the square brackets $([\cdot])$ denote the mass concentration of the ICG complex, excipient, or PS-block in the THF stream [the PS-block is the $1.6 \mathrm{k}$ molecular weight (MW) block of the $1.6 \mathrm{k}-5 \mathrm{k}$ PS- $b$-PEG copolymer]. Alternatively, the dye core loading can be described on the basis of the ICG alone

ICG core loading

$$
=\frac{[\mathrm{ICG}]}{[\mathrm{ICG}]+[\text { counter-ion }]+[\text { excipient }]+[\text { PS-block }]} \text {. }
$$

Equation (2) is relevant when encapsulation efficiency is studied by changing the ICG:counter-ion ratio.

\subsection{ICG NC Loading Limits}

While studying ICG-C $\mathrm{C}_{8}$ complex NC stability with either VE or PS cores, we observed that, at high ICG complex loadings, a glitter-like precipitate formed in the solutions several hours after NC formation. The precipitates were not NC flocculated particles, but rather an ICG crystal phase. The precipitates could be filtered out with a $5-\mu \mathrm{m}$ filter, and the resulting filtrate contained a single NC population. By measuring the size change and ICG concentration change by absorbance, we determined that each type of $\mathrm{NC}$ core had a certain loading threshold above which ICG complex precipitates would form and below which no precipitates would form and NCs would be stable for weeks to months (see Fig. 3).

In the case of PS cores, NCs loaded with $4.1 \%$ and $8.7 \%$ core loading ICG- $\mathrm{C}_{8}$ complex were stable, but those loaded at $38 \%$ dropped to a final loading of $9.8 \%$ within a few hours. In the case of VE cores, NCs loaded at $32.9 \%, 58.9 \%$, and $77.7 \%$ all dropped to final effective loadings of $31.6 \%, 30.5 \%$, and $27.8 \%$, respectively. Further formulations with VE below this threshold were all stable (no precipitate formed). In the case of the ICG complex loaded into PS- $b$-PEG micelles (no cosolute), NCs loaded at $78 \%$ dropped to a final loading of about $42 \%$. These measurements indicate that the ICG complex is 
(a)

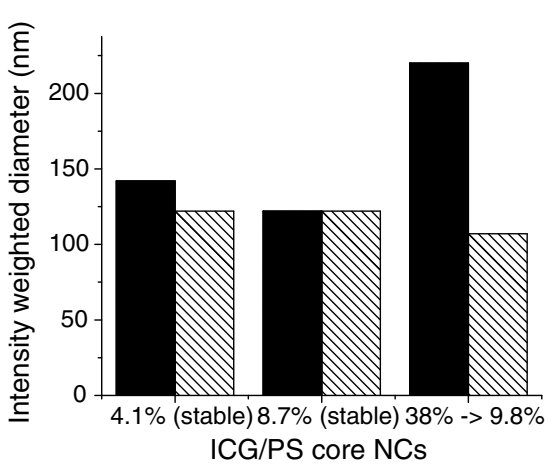

(b)

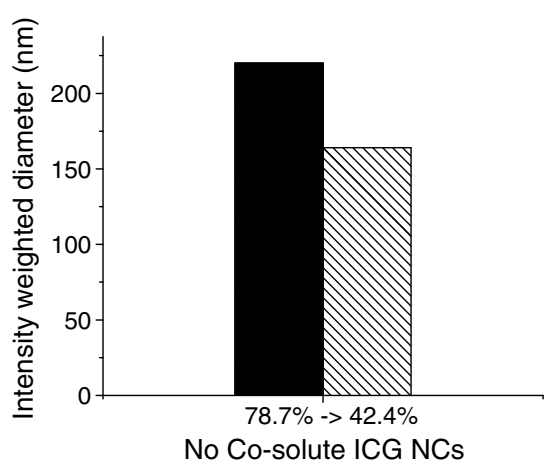

(c)

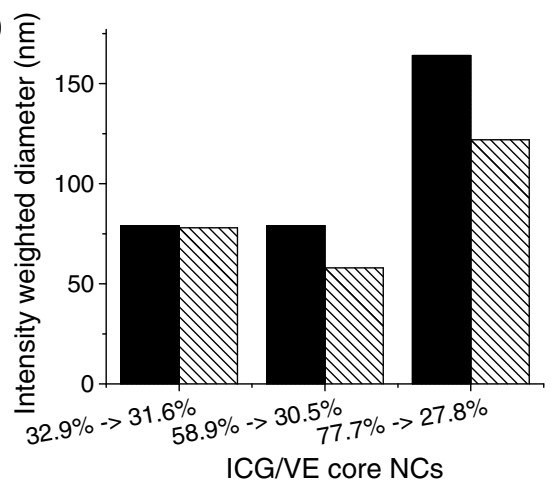

Fig. 3 NC size change following precipitation of ICG-C 8 with different core materials and corresponding changes in ICG complex core loading shown at the abscissa: (a) PS core, (b) no cosolute, (c) VE core (solid bars, preprecipitation; striped bars, postprecipitation). The maximum stable loading levels are dependent on the core material.

less compatible with PS than with VE. Since 10 wt. \% ICG complex core loading maximizes FL, and since we had experience with $\mathrm{VE}$ as a coexcipient for therapeutics in the $\mathrm{NC}$ core, we studied only VE core NCs for subsequent experiments. VE core NCs stabilized by PS- $b$-PEG were found to be size-stable for more than 6 months (data not shown) as long as the ICG loading was below the limits shown in Fig. 3.

\subsection{Size Control and Fluorescence Scaling of ICG NCs}

Since NC size is an important design parameter, we demonstrate that these ICG NCs could be produced at different sizes at a constant ICG complex loading (the optimal FL loading determined earlier). The NC size produced by FNP primarily depends on the solution concentration. ${ }^{46}$ We held the ratio of all components constant and varied the total solids from 1 to $100 \mathrm{mg} / \mathrm{mL}$ in the feed stream. The size distributions [shown in Fig. 4(a)] become slightly broader at larger sizes, in accordance with mechanism of growth in FNP. ${ }^{52}$ The NC size scales linearly with the total solids concentration up until $70 \mathrm{mg} / \mathrm{mL}$, at which point the size plateaus at $\sim 180 \mathrm{~nm}$ [Fig. 4(b)].

The per-particle FL [Fig. 4(c)] scales linearly with the core volume, i.e., the core diameter to the third power: $\frac{\mathrm{FL}}{\mathrm{NP}}=$ $\left(5.01 \times 10^{-14}\right)\left(D^{2.98}\right), R^{2}=0.98$. This shows that the ICG is encapsulated uniformly in the core of the NCs and not just at the core-shell interface, which would have a $D^{2}$ scaling. As a result, larger particles are more fluorescent at constant dye loading. We hypothesize that previous studies that loaded ICG into preformed NCs through the aqueous phase would produce NCs with ICG at the NC interface. However, in those studies FL versus size was not reported, so the localization of the dye via these other assembly processes cannot be confirmed.

\subsection{Encapsulation Efficiency and lon Pairing}

Previously, we had successfully created NCs by forming hydrophobic ion pairs of therapeutic drugs using the in situ complexation of anionic and cationic compounds when one was solubilized in the aqueous phase and one in the organic phase. ${ }^{53,54}$ To test the ability to make ICG NCs by a similar ion pairing, the ratio of TOAC to ICG was varied and the degree of ICG encapsulation [Fig. 5(b)] was measured with UV-VIS absorbance. The sizes of the NCs were held constant [Fig. 5(a)] except for the micelle sample, which had no VE and no TOAC. However, the ICG core loading was not kept constant across samples. Since the TOAC:ICG ratio was varied, and in some cases no TOAC was used, the ICG core loading [Eq. (2)] was kept in a range from $2.5 \%$ to $25 \%$. For reference, an ICG complex core loading at the optimal 10 wt. \% (with $1: 1$ ICG:TOAC) is equivalent to an ICG core loading at $6.5 \mathrm{wt} . \%$. These data are tabulated in Table 3.

For a 1:1 or higher ratio of TOAC:ICG, the cationic counter-ion drives essentially complete encapsulation of the ICG into the NC core. However, at only $10 \mathrm{wt}$. \% or less ICG complex core loading, the preponderance of hydrophobic VE and PS-chains in the core still substantially captures the ICG during rapid precipitation. The $0: 10,1: 10$, and $1: 2$ ratios of TOAC:ICG yield $94 \%, 89 \%$, and $97 \%$ ICG encapsulation, 

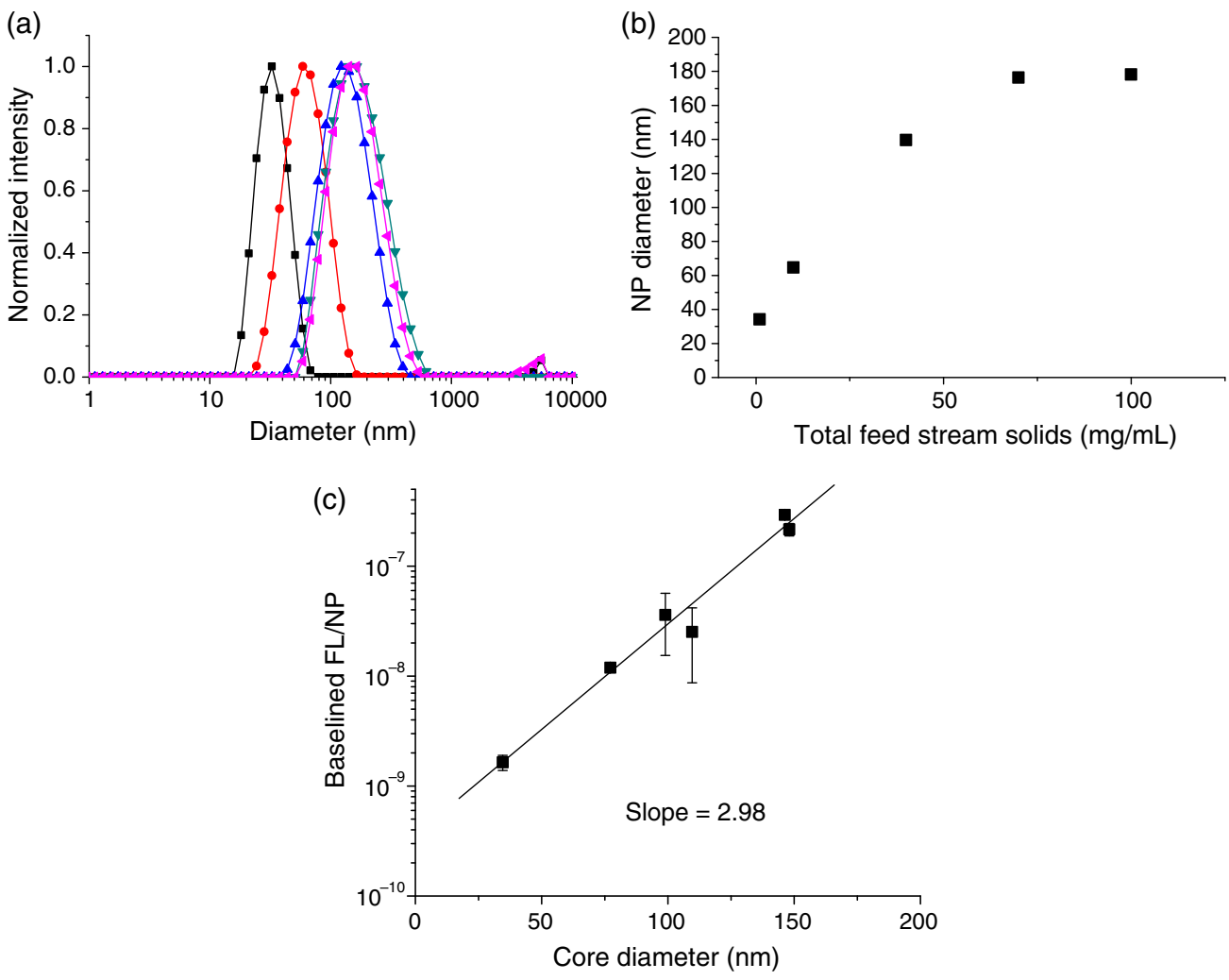

Fig. 4 (a) Size distributions for ICG-TOAC (ICG-C $)$ in situ formed NCs $(-1 \mathrm{mg} / \mathrm{mL}$, $10 \mathrm{mg} / \mathrm{mL}$, $40 \mathrm{mg} / \mathrm{mL}, \nabla 70 \mathrm{mg} / \mathrm{mL}, 4100 \mathrm{mg} / \mathrm{mL}$ ), (b) NC sizes versus total solids concentration. (c) FL intensity per NC versus core diameter. The FL per NC (intrinsic NC FL) scales linearly with the core diameter to the third power.

respectively. PS- $b$-PEG and ICG (no TOAC and no VE) gave encapsulation at $97 \%$ in the $25-\mathrm{nm}$ micelle NCs. DeVoiselle et al. $^{26}$ investigated the complexation of ICG in the presence of various surfactants using tensiometric and FL measurements and found that amphiphilic ICG assembles at interfaces. Kircherr et al. studied the encapsulation of ICG monosodium salt within nonionic surfactant micelles and achieved $90 \%$ to 95\% loading efficiency but only $61 \%$ within Pluronic F68 micelles due to its higher hydrophilicity. ${ }^{28}$ Similarly, Kim et al. ${ }^{27}$ achieved encapsulation efficiencies of $39 \%$ to $79 \%$ for hydrophobic ICG-tetrabutylammonium salts in Pluronic F127. Rodriguez et al. ${ }^{29}$ formed hydrophobic salts of ICG with tetrabutylammonium iodide (TBA) within poly(styrene)- $b$-poly(styrene-alt-maleic anhydride) micelles and achieved $87 \%$ encapsulation efficiency. The rapid precipitation by FNP yielded higher ICG encapsulation efficiencies than any of the previously reported loading techniques. Since the previous techniques involved introducing the ICG from or through the aqueous phase, the lack of uniform encapsulation into the core may be the reason. The previous studies could not vary NC size, as we can with FNP, so no comparison of FL intensity with size or loading efficiency is possible.

We calculated the available interfacial surface area available for ICG adsorption in the case of the polymeric micelles, which from Fig. 5(a) is $25 \mathrm{~nm}$ in diameter. Based on the projected surface area of an ICG molecule $\sim 0.15 \mathrm{~nm}^{2}$ and molecular volume of $5.8 \times 10^{-2} \mathrm{~nm}^{3}$ (calculated with ChemAxon MarvinSketch v6.2.2 Geometry Plugin), ${ }^{55}$ a micelle could accommodate $13 \times$ $10^{3}$ ICG molecules on its surface and $141 \times 10^{3}$ ICG molecules in the core. Since the ICG loading in the micelle core is $\sim 25$ wt. $\%$, the core would contain $\sim 35 \times 10^{3}$ ICG molecules. Therefore, we would expect ICG to be loaded both in the core and at the interface, which might be the reason for the very efficient loading of the micelles, even in the absence of the TOAC counter ion. The surface loading would become less significant for larger NCs.

\subsection{ICG NC-BSA Stability}

It is important that the dye remain trapped in the NC core, so the NCs can be effectively tracked without fluorescent signal being confounded with free dye or dye bound to proteins. Kim et al. ${ }^{27}$ reported Pluronic F127 micelles loaded with ICG-TBA salt $\left(\mathrm{ICG}-\mathrm{C}_{4}\right)$ and incubated with $50 \%$ serum. A threefold increase in FL was seen due to the partitioning of ICG from the micelles. However, we have previously demonstrated that the relatively low CMC of these PPO-PEO copolymers comprising Pluronic F127 enables them to serve as "molecular shuttles" of hydrophobic components in the cores of NCs. ${ }^{5}$ The block copolymers used in FNP are chosen to have hydrophobic blocks large enough to not partition off the NC surface; they are kinetically frozen. ICG is known to bind strongly to serum proteins, in particular, albumin, globulins, and $\alpha$-lipoproteins. ${ }^{24,56-59}$ As noted above, Rodriguez et al. tested the release of ICG-tetrabutylammonium complex $\left(\mathrm{ICG}-\mathrm{C}_{4}\right)$ from $\mathrm{NCs}$ only in DI water. This does not address the stability of ICG in NCs in vivo because in vivo ion exchange of the ICG- $\mathrm{C}_{4}$ and partitioning to serum proteins can occur, as seen by Kim. Zerda et al. ${ }^{53}$ created carbon nanotube-ICG constructs where ICG molecules were attached to carbon nanotubes simply by $\pi-\pi$ hydrophobic 

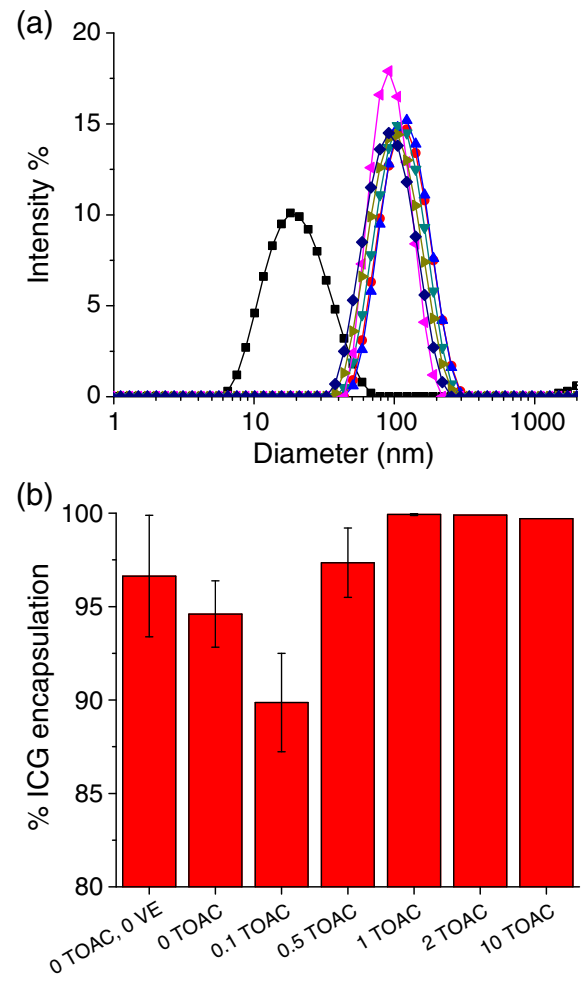

Fig. 5 ICG encapsulation efficiencies as a function of TOAC:ICG ratio (in situ complex formation): (a) size distributions of particles (- micelles (0 TOAC, 0 VE), $\bullet 0$ TOAC, ^ 1:10 TOAC:ICG, $\checkmark$ 1:2 TOAC:ICG, \ 1:1 TOAC:ICG, 2:1 TOAC:ICG, 10:1 TOAC:ICG) and (b) the ratio of TOAC to ICG was varied from 0 to 10:1 for in situ ICG NC precipitation. The number of TOAC equivalents relative to ICG is labeled on the abscissa.

interactions. They tested the stability of these complexes by incubation in $10 \%$ serum $/ 90 \%$ PBS for $24 \mathrm{~h}$, which gives an albumin concentration of only $0.4 \mathrm{wt}$. \%, 10 times lower than physiological levels. Even under these mild conditions, optical absorbance increased by $25 \%$, which indicates the partitioning to BSA. Our objective was to determine if the ICG: counterion complex trapped in NC cores was stable in the presence of physiological levels of serum protein (albumin) and if the more hydrophobic octylammonium $\left(\mathrm{C}_{8}\right)$ and dodecylammonium $\left(\mathrm{C}_{12}\right)$ counter-ions might prevent ion exchange and make the NC less susceptible to partitioning to serum proteins.

$\mathrm{NCs}$ formed from ICG and tetrabutylammonium $\left(\mathrm{C}_{4}\right)$ in situ, tetraoctylammonium $\left(\mathrm{C}_{8}\right)$ in situ and premade, and tetradodecylammonium $\left(\mathrm{C}_{12}\right)$ in situ complexes were incubated at room temperature with 4 wt. $\%$ BSA in $5 \mathrm{mM}$ phosphate buffer at $\mathrm{pH} 7.0$, and the change in FL was measured over a period of $\sim 2 \mathrm{~h}$. The change in total system FL demonstrates ion exchange of ICG from the NC core to albumin, even when protected in the hydrophobic NC core.

The intrinsic FL of ICG in buffer and the FL of ICG in serum protein were first measured, and the data are shown in Fig. 6(a). The intrinsic FL, as defined by the slope of FL versus [ICG], is $\sim 1.6 \times$ higher for ICG bound to protein than for ICG in buffer, which is consistent with the data in Fig. 1(f) (the correlation fits are shown in Fig. 6). Similarly, the intrinsic FL slopes of the various ICG NC systems were measured, and representative data for ICG-C $\mathrm{C}_{8}$ in situ are shown in Fig. 6(b). NC intrinsic FL was found to be 1 to 2 orders of magnitude lower than that for free ICG in buffer. The increase in FL upon ICG binding to $\mathrm{BSA}$ is due to a combination of (1) molecular disaggregation and stabilization of the monomeric ICG on the BSA, ${ }^{41,42}$ (2) reduction of FRET within each NC core, ${ }^{46,51}$ and (3) "rigidization" of the bound ICG, which reduces dissipative degrees of freedom such as molecular vibration and rotation, resulting in an increase in optical emission. ${ }^{41,42}$ The relative impacts of these effects on observed FL can be estimated from Fig. 1(f), which shows a $1.6 \times$ increase in FL for free ICG binding to albumin due to an increase in QY. Philip et al. ${ }^{41}$ have reported a FL QY increase from $2.7 \%$ to $4.0 \%$ for ICG bound to albumin, confirming this measurement. Furthermore, FL is reduced by a factor of $2.8 \times$ by complexing and encapsulating free ICG within $\mathrm{NC}$ cores, due to a combination of aggregation and FRETinduced quenching. The strong tendency of ICG to form stable, self-quenching aggregates at low concentrations suggests that aggregation would have a larger impact on FL than FRET. ${ }^{24,41,60}$

Experiments with the various $\mathrm{NC}$ formulations show the increase in FL as the ICG-ion pairs exchange from the NCs onto BSA in solution at $4 \mathrm{wt}$ \%, which is at the physiological

Table 3 ICG encapsulation efficiency, ICG core loading and equivalent complex core loading, and NC diameters for the encapsulation efficiency study in Fig. 6, where the ICG:TOAC ratio was varied. The VE concentration was held constant at $8.9 \mathrm{mg} / \mathrm{mL}$ in the THF stream except for the micelle case (OTOAC, OVE) where no VE was used. VE constituted $40 \%$ to $48 \%$ of the total NC mass.

\begin{tabular}{lcccc} 
Sample & Encapsulation efficiency (\%) & ICG core loading (wt. \%) & Complex core loading (wt. \%) & Diameter $(\mathrm{nm})$ \\
\hline OTOAC/OVE (micelle) & 96.6 & 24.8 & N/A & N/A \\
OTOAC & 94.6 & 6.30 & 3.80 & 120 \\
0.1 eqv. TOAC & 89.9 & 18.3 & 10.3 & 120 \\
0.5 eqv. TOAC & 97.4 & 12.0 & 11.0 & 113 \\
1 eqv. TOAC & 99.9 & 7.00 & 10.9 & 100 \\
2 eqv. TOAC & 99.9 & 6.90 & 3.90 & 109 \\
10 eqv. TOAC & 99.7 & 2.50 & 100 \\
\hline
\end{tabular}

a Defined by Eq. (2)-takes ICG encapsulation efficiency into account and assumes excess TOAC is not incorporated into the core.

${ }^{b}$ The complex core loading calculation assumes a 1:1 ICG:TOAC pairing and, thus, the limiting reagent defines the loading. The complex core loading is not defined for systems with no counter-ion (OTOAC). 
(a)

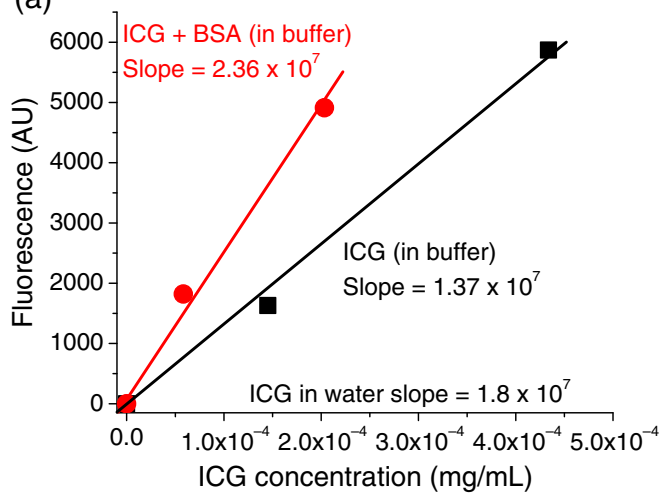

(b)

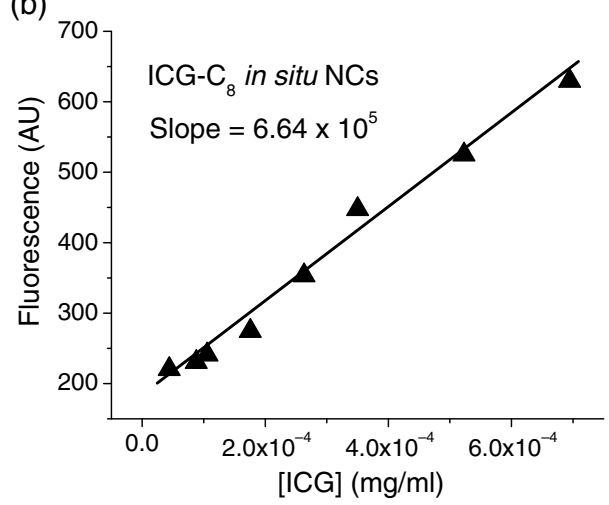

Fig. 6 (a) FL transfer experiments showing the increase in intrinsic ICG FL (slope) upon the addition of $\mathrm{BSA}$ to ICG dissolved in $5 \mathrm{mM}$ phosphate buffer. For pure ICG, the regression fit is: $\mathrm{FL}=1.37 \times 10^{7}[\mathrm{ICG}(\mathrm{mg} / \mathrm{mL})], \quad R^{2}=0.996$, for $\mathrm{ICG}+\mathrm{BSA}: \quad \mathrm{FL}=2.36 \times 10^{7}[\mathrm{ICG}(\mathrm{mg} / \mathrm{mL})]$, $R^{2}=0.991$. (b) Intrinsic FL of ICG- $\mathrm{C}_{8}$ in situ, which is an order of magnitude lower than the intrinsic $\mathrm{FL}$ of free ICG solution and ICG + BSA solution. ICG- $\mathrm{C}_{8}$ complex loaded at $10 \mathrm{wt}$. \% core loading.

protein concentration. ${ }^{61}$ In the time course FL measurements, shown in Fig. 7, the ICG-C $\mathrm{C}_{4}$ in situ formulation displays the largest $(\sim 800 \%)$ rise in FL due to a large amount of ICG dye ion exchanging and partitioning out of the $\mathrm{NC}$ core and binding to the albumin. Both the $\mathrm{C}_{8}$ in situ and premade formulations display a relatively small change in FL, indicating a small amount partitions out since it is more hydrophobic than the $\mathrm{C}_{4}$ formulation. Studies with free ICG incubated with 4 wt. \% BSA showed that the FL went immediately to the value shown in Fig. 1(f) and stayed there over the course of $120 \mathrm{~min}$. Surprisingly, the $\mathrm{C}_{12}$ counter-ion does not confer the best ICG stability, having a FL change intermediate between the $\mathrm{C}_{4}$ and $\mathrm{C}_{8}$ counter-ions. This is likely due to (1) the $\mathrm{C}_{12}$ counter-ion micellizing more readily than the $\mathrm{C}_{8}$, thus providing a "molecular shuttling" effect that transfers additional ICG to the BSA. This effect has been observed in similar NC systems previously. ${ }^{5}$ A secondary effect may arise from specific favorable interactions between the $\mathrm{C}_{12}$ counter-ion and residues in the BSA.

By combining the data in Figs. 6 and 7, an estimate of the fraction of ICG transferred from NCs to BSA can be obtained

$$
\begin{aligned}
@ t_{0}: \mathrm{FL}_{i}= & \text { slope }_{\mathrm{ICG} / \mathrm{NC}} \times[\mathrm{ICG}]_{\mathrm{NC}, i}, \\
@ t_{\infty}: \mathrm{FL}_{f}= & \text { slope }_{\mathrm{ICG} / \mathrm{BSA}} \times[\mathrm{ICG}]_{\mathrm{BSA}}+\text { slope }_{\mathrm{ICG} / \mathrm{NC}} \\
& \times[\mathrm{ICG}]_{\mathrm{NC}, f} .
\end{aligned}
$$

By combining Eqs. (3) and (4), the fraction of ICG associated with BSA can be estimated

$$
\frac{C_{\mathrm{ICG} / \mathrm{BSA}}}{C_{\mathrm{ICG}, \text { total }}}=\left(\frac{\mathrm{FL}_{f}-\mathrm{FL}_{i}}{\mathrm{FL}_{i}}\right)\left(\frac{\text { slope }_{\mathrm{ICG} / \mathrm{NC}}}{\text { slope }_{\mathrm{ICG} / \mathrm{BSA}}-\text { slope }_{\mathrm{ICG} / \mathrm{NC}}}\right),
$$

where $\mathrm{FL}_{i}$ and, $\mathrm{FL}_{f}$ are the initial fluorescence and final fluorescence (AU), respectively, $C_{\mathrm{ICG} / \mathrm{BSA}}$ is the concentration of ICG associated with BSA $(\mathrm{mg} / \mathrm{mL}), C_{\mathrm{ICG} \text {,total }}$ is the total concentration of ICG in the suspension $(\mathrm{mg} / \mathrm{mL})$, slope $_{\mathrm{ICG} / \mathrm{NC}}$ is the FL slope of ICG in NCs (Table 4), and slope ICG $/ \mathrm{BSA}_{\text {is }}$ the FL slope of ICG associated with BSA (see Fig. 6).

Table 4 summarizes the ICG NC-BSA stability data. There is a positive correlation between the change in FL and the fraction of ICG transferred from NC to BSA. We see that the $\mathrm{C}_{4}$ counterion formulation provides little stability to the ICG, with $\sim 62 \%$ of the ICG transferring to BSA, in agreement with the large FL rise in Fig. 7. The $\mathrm{C}_{8}$ complex formed either in situ or premade provides the best stability, with only $3 \%$ to $4 \%$ of the ICG transferred away. The $\mathrm{C}_{12}$ complex formed in situ has lower stability than the $\mathrm{C}_{8}$ complex but is significantly more stable than the $\mathrm{C}_{4}$.

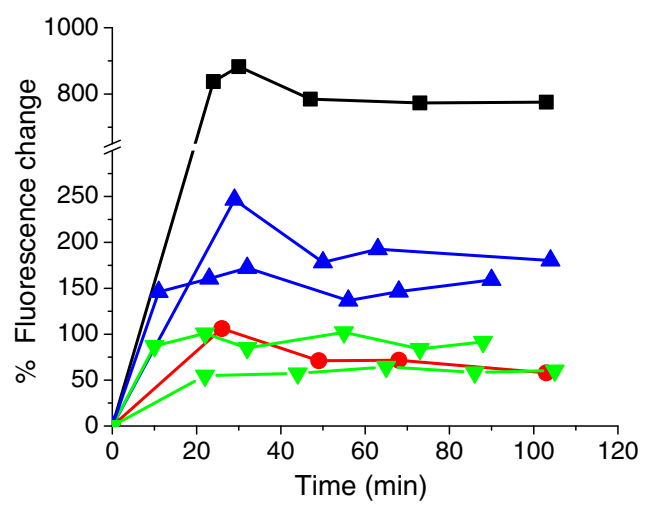

Fig. 7 Percent FL change of various ICG NC formulations in the presence of $5 \mathrm{mM}$ phosphate and $4 \mathrm{wt} . \%(40 \mathrm{mg} / \mathrm{mL})$ BSA. [- $\mathrm{C}_{4}$ in situ complex, $\bullet \mathrm{C}_{8}$ in situ complex, $\boldsymbol{\nabla} \mathrm{C}_{8}$ premade complex (two replicates shown), $\Delta \mathrm{C}_{12}$ in situ complex (two replicates shown)]. The $\mathrm{C}_{4}$ complex showed the greatest change in FL indicating the greatest amount of ICG exchanged away, as expected, while the $\mathrm{C}_{8}$ counter-ion provided the best protection.

Table 4 Summary of ICG NC-BSA stability data.

\begin{tabular}{lccc}
\hline Sample & $\begin{array}{c}\text { Intrinsic } \\
\mathrm{FL}(\text { slope })\end{array}$ & $\begin{array}{c}\text { Change in } \\
\mathrm{FL}(\%)\end{array}$ & $\begin{array}{c}\text { ICG transfer (\%) } \\
(\mathrm{NC} \rightarrow \mathrm{BSA})\end{array}$ \\
\hline ICG-C $_{4}$ in situ & $7.25 \times 10^{6}$ & $778 \pm 6.1$ & 62 \\
ICG-C $_{8}$ in situ & $6.64 \times 10^{5}$ & $67 \pm 7.8$ & 3 \\
ICG-C $_{12}$ in situ & $2.38 \times 10^{6}$ & $166 \pm 20$ & 19 \\
ICG-C $_{8}$ premade & $1.17 \times 10^{6}$ & $76 \pm 12$ & 4 \\
\hline
\end{tabular}




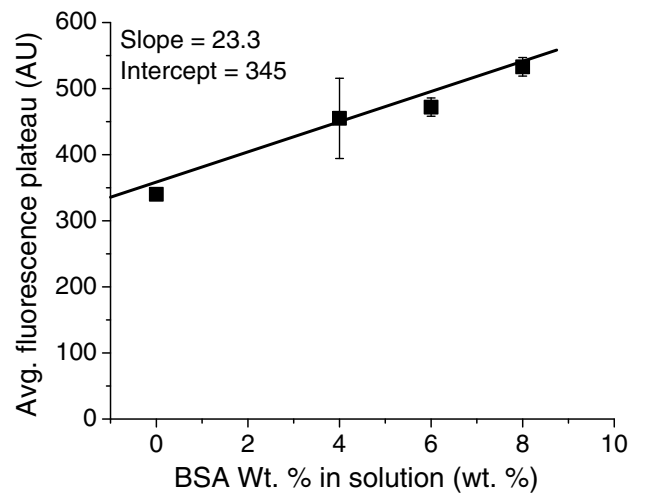

Fig. 8 Average FL plateau height versus wt. \% BSA in solution for ICG- $\mathrm{C}_{8}$ in situ formed NCs, which was the most stable formulation tested. A linear dependence is apparent with slope $=23.3$, intercept $=345$. This shows that the ICG partitions out of the NC core to an extent dependent on the size of the external BSA sink.

When ICG partitions from NC cores to BSA, there is a rise in the intrinsic FL slope (Fig. 6) that can be 1 to 2 orders of magnitude higher. Thus, even a small amount of BSA-bound ICG can lead to a large change in FL signal. While the ICG- $\mathrm{C}_{8}$ and $-\mathrm{C}_{12} \mathrm{NC}$ formulations are stable in phosphate buffer, when given the sink of the BSA protein, ICG is rapidly exchanged away from the NC. Clearly, this would be problematic for quantification in in vivo studies.

Finally, we sought to verify that $4 \mathrm{wt} . \%$ (40 mg/mL) BSA, which is the physiological albumin concentration, ${ }^{61}$ was in sufficient excess to represent a true sink for the ICG. In these experiments, the BSA:ICG molar ratio was $\sim 10: 1$, but, if an ICG NC suspension is injected into a mouse (blood volume $1.5 \mathrm{~mL}$ ) or human (blood volume $5 \mathrm{~L}$ ), the total molar ratio of BSA to ICG would be much higher, which can result in ICG being continuously drawn out from NC cores. Therefore, we incubated the most stable NC formulation tested (ICG-C ${ }_{8}$ in situ complex) with 4,6 , and 8 wt. \% BSA and measured the FL time course over $\sim 2 \mathrm{~h}$. The results are shown in Fig. 8, where we see a dependence of the final FL plateau on the BSA concentration. With a greater external sink of BSA, the amount of ICG exchanged from the NC is greater.

\section{Conclusions}

In this work, we report the formulation of ICG-based NCs, formed either by preforming an oil soluble (hydrophobic) ICG-TOAC or ICG-TDDAC ion pair or by in situ forming the ion pair and NC in a one-step process using FNP. Both methods yield similar NCs in terms of size, stability, optical absorption, and FL for each type of counter ion. Maximum FL-perparticle for the ICG-TOAC complex is $10 \mathrm{wt}$ \% core loading, which represents the balance between increasing the concentration of dye in a particle and minimizing FL quenching due to molecular aggregation and Förster energy transfer at high loadings. To elucidate the mechanism of the ICG complex formation and NC loading, we varied the ratio of TOAC to ICG and showed that ICG encapsulation efficiencies of $100 \%$ were achieved by using a 1:1 or higher ratio of cationic counter ion to ICG. However, the rapid precipitation process produces relatively high encapsulation efficiencies (greater than 80\%) even without stoichiometric counter-ion ratios. Limits for the ICG loading of the NCs are presented based on the type of core material used. Above these limits, ICG complex precipitates outside the NCs into large crystals. VE is found to be a more accommodating core material compared to $1.5 \mathrm{k}$ PS. We also demonstrate the control of NC size between 30 and $180 \mathrm{~nm}$ at constant ICG loading and show that the intrinsic FL of the NCs scale linearly with the volume of the NC core.

Finally, we present data showing the photostability of ICG $\mathrm{NCs}$ in the presence of physiologically relevant amounts of albumin, for which ICG has a very strong binding affinity. The data on ion exchanging of ICG from NC cores provide important data currently lacking in the literature.

We show that the type of counter-ion used to form the hydrophobic ICG complex greatly affects its stability in the presence of BSA, with tetrabutylammonium $\left(\mathrm{C}_{4}\right)$ being the worst $(62 \%$ loss), tetraoctylammonium $\left(\mathrm{C}_{8}\right)$ being the best (3\% to $4 \%$ loss), and tetradodecylammonium $\left(\mathrm{C}_{12}\right)$ being intermediate $(19 \%$ loss). The loss of ICG from the NC would still leave a 100$\mathrm{nm} \mathrm{NC}$ with at least $10^{4}$ ICG molecules in the core-indicating that a bright FL signal would still be achieved. However, the instability must be considered when attempting to relate FL images to NC concentrations in applications such as targeting studies in vitro and in vivo.

\section{Experimental Section}

\subsection{Materials}

Two forms of ICG were used: Cardiogreen, which contains NaI, was obtained from Sigma-Aldrich (I2633) and an iodine-free ICG was obtained from Persis Science LLC in Princeton, New Jersey. The cationic complexation agents TBA (86890), tetrabutylammonium chloride (86870), TOAC (87991), and TDDAC (87250) were obtained from Sigma-Aldrich and used without modification. Hydroxyl terminated poly(styrene) and poly(styrene)- $b$-poly(ethylene glycol) $(1.5 \mathrm{k}-b-5 \mathrm{k})$ were synthesized as previously described. ${ }^{62}$ Poly(styrene)- $b$-poly(ethylene glycol) (1.6k- $b-5 \mathrm{k})$ was obtained from Polymer Source (P13141-SEO, Polymer Source, Montreal, Canada). High-pressure liquid chromatography (HPLC) grade THF and diethyl ether (DEE) were obtained from Fisher Scientific (T425-4 and AC615080010, respectively, Fisher Scientific), and ultrapure water was obtained from a Barnstead NanoPure system delivering $>17.8 \mathrm{M} \Omega-\mathrm{cm}$ (hereafter referred to as "Milli-Q water").

\subsection{Methods}

\subsubsection{Ion pairing ICG complexation reaction}

$193.70 \mathrm{mg}$ of iodine-free ICG (sodium 4-[2[(1E,3E,5E, 7Z(-7[1,1-dimethyl-3(4-fultonatobutyl)benzo(e)indol-2-ylidene] hepta-1,3,5-trienyl]-1,1-dimethylbenzo(e)indol-3-ium-3-yl \}butane1-sulfonate, formula corresponding to $\mathrm{C}_{43} \mathrm{H}_{47} \mathrm{~N}_{2} \mathrm{NaO}_{6} \mathrm{~S}_{2}$, MW 774.96) was placed in $250 \mathrm{~mL}$ of dried DEE in a $1 \mathrm{~L}$ polypropylene container. This mixture was allowed to mix under nitrogen for $30 \mathrm{~min}$. To this mixture was added $251.2 \mathrm{mg}$ of TOAC, while mixing under nitrogen. While continuously mixing, $250 \mathrm{~mL}$ of Milli-Q water was added. The container was sealed with a gasket and lid and placed into a Flacktek DAC400 Speedmixer for $10 \mathrm{~min}$ using the following settings: $2 \mathrm{~min}$ at $800 \mathrm{RPM}, 2 \mathrm{~min}$ at $1200 \mathrm{RPM}, 4 \mathrm{~min}$ at $1800 \mathrm{RPM}$, and finally $2 \mathrm{~min}$ at $2000 \mathrm{RPM}$. The mixture was removed from the Speedmixer and observed to determine that all of the green color (ICG) was removed from the bottom aqueous layer and dissolved as a dark green color in the top (DEE) layer. The reaction mixture was placed into a $1 \mathrm{~L}$ separation funnel and shaken 
vigorously for $1 \mathrm{~min}$. The funnel was allowed to stand for $15 \mathrm{~min}$, while occasional spinning of the apparatus was performed to collect all the water droplets. After $5 \mathrm{~min}$, the bottom layer was drained and another $250 \mathrm{~mL}$ of Milli-Q water was added and the same procedure was repeated three times. The ether solution was stirred by hand and 20 grams of anhydrous magnesium sulfate was added and shaken slightly. The magnesium sulfate was removed by threefold filtration. The aqueous phase appeared colorless, and the ether layer was placed into a 500-mL, single neck round bottom flask. The ether was removed by rotary evaporation. When there was a final solution amount of $\sim 10$ to $12 \mathrm{~mL}$, it was transferred to a small $20 \mathrm{~mL}$ vial and placed on the rotary evaporator under vacuum at $40^{\circ} \mathrm{C}$ for about $45 \mathrm{~min}$. The resulting crystalline material was dried at room temperature under high vacuum overnight. The material was found to be very insoluble in water. $286.7 \mathrm{mg}(94.24 \%$ yield) was recovered after vacuum drying. The material was dissolved at $1.0 \mathrm{mg} / \mathrm{mL}$ in acetonitrile and run through HPLC on a $\mathrm{C}_{18} 5-\mu \mathrm{m}$ column using $35 / 65$ acetonitrile:water at a flow rate of $1.75 \mathrm{ml} / \mathrm{min}$. Only one peak was observed at $2.27 \mathrm{~min}$. The DAD, FL, and mass indicated the presence of only the ICG-tetraoctylammonium complex with $1: 1$ molar pairing having a mw of 1218.86 (name: tetraoctylammonium 4-[2-[1E,3E,5E,7Z)-7-[1,1-dimethyl-3-(4-sulfonatobutyl) benzo(e)indol-2-ylidene]hepta-1,3,5-trienyl]-1,1-dimethylbenzo[e]indol-3-ium-3-yl]butane-1-sulfonate, the formula corresponding to $\mathrm{C}_{75} \mathrm{H}_{115} \mathrm{~N}_{3} \mathrm{O}_{6} \mathrm{~S}_{2}$ ). This was confirmed by proton and carbon NMR as well as FL using excitation at $785 \mathrm{~nm}$ and measuring emission at 800 to $1300 \mathrm{~nm}$. Excitation and emission curves were also calculated. The UV-Vis showed a sharp peak at a max of $794 \mathrm{~nm}$ and purity by elemental analysis of $>98 \%$.

\subsubsection{ICG NC formation in situ (fluorescence-per-particle determination)}

The optimal NC loading of ICG for maximum FL on a perparticle basis was determined by formulating particles with different core loadings of ICG. ICG concentrations from 0.3 to $8.8 \mathrm{mg} / \mathrm{mL}$ in water were mixed against a THF stream containing 1 molar equivalent of TOAC and VE from 1 to $15.5 \mathrm{mg} / \mathrm{mL}$. PS- $b$-PEG was present in the THF stream from 9.3 to $12.8 \mathrm{mg} / \mathrm{mL}$. Total solids ranged from 17.9 to $28.3 \mathrm{mg} / \mathrm{mL}$ and NC sizes were in the range from 100 to $160 \mathrm{~nm}$. ICG complex core loadings were varied between 0.014 and 78 wt. $\%$. THF and water streams were mixed in a $1: 1$ volume ratio in a confined impinging jets (CIJ) mixer and quenched in 9 parts water (overall 1:10 mixing ratio). The resulting NCs were characterized for size by dynamic light scattering (DLS) and diluted to multiple concentrations for FL measurements to establish the linearity of FL versus [NC]. The slope of the FL versus [NC] or [ICG] line was recorded as the intrinsic FL of that NC system. The system with the highest intrinsic FL (equivalent to per-particle FL) was the optimally loaded system.

\subsubsection{ICG formation in situ (10 wt. \% core loading)}

ICG was dissolved in water at $0.80 \mathrm{mg} / \mathrm{mL}$. TOAC was dissolved in THF at $0.52 \mathrm{mg} / \mathrm{mL}$, PS- $b$-PEG at $9.7 \mathrm{mg} / \mathrm{mL}$, and $\mathrm{VE}$ at $8.2 \mathrm{mg} / \mathrm{mL}$ (so that VE plus TOAC was 1 wt. \% in the THF feed stream). The aqueous and organic feed streams were rapidly mixed in equal volumes in a CIJ mixer according to Johnson and Prud'homme ${ }^{43}$ to form the hydrophobic
ICG-TOAC complex and polymer-protected NCs via FNP in one step.

\subsubsection{In situ ICG encapsulation efficiency-variation of ICG: TOAC ratio}

ICG NCs were prepared with varying ratios of ICG:TOAC to test the required amount of TOAC for stable ICG complex and NC formation. TOAC:ICG ratios of $1: 10,1: 2,1: 1,2: 1$, and 10:1 were tested. Control experiments with no TOAC, and no TOAC and no VE, were also performed. The VE concentration was held constant at 1 wt. $\%(8.9 \mathrm{mg} / \mathrm{mL}$ in THF $)$, [ICG] ranged from 0.3 to $3 \mathrm{mg} / \mathrm{mL}$ in the water feed, and [PS- $b$-PEG] was equal to $[\mathrm{ICG}]+[\mathrm{TOAC}]+[\mathrm{VE}]$ on a weight basis. The THF stream and ICG-in-water stream were rapidly mixed in the CIJ mixer and quenched in a water reservoir with a volume of 9 times that of the water volume being mixed. Therefore, the final mixing ratio of THF: water was $1: 10$. The NC sizes were measured with DLS. The samples formed were centrifuge filtered with Amicon 100k MWCO filters (UFC210024, EMD Millipore, Billerica, Massachusetts) at $7000 \mathrm{~g}$ for $20 \mathrm{~min}$ to separate the free ICG from NCs. Free ICG concentrations in the filtrates were measured by optical absorbance (Evolution 300 UV-Vis-NIR spectrophotometer, Thermo Fisher Scientific, Bridgewater, New Jersey) and compared against initial absorbance values for the initial NC suspension to determine the free ICG\% and hence the encapsulation efficiency.

\subsubsection{ICG NC size control experiments}

ICG NCs were prepared with varying amounts of total solids but a constant ratio of all solid components. Total NC solids in the feed ranged from 1 to $100 \mathrm{mg} / \mathrm{mL}$. ICG loading was held constant with 1:1 ICG:TOAC and 10.5\% ICG complex core loading. By mass, the NCs were $43 \%$ VE, $4 \%$ ICG, $3 \%$ TOAC, and $50 \%$ PS- $b$-PEG, the same as the optimally fluorescent formulations determined earlier. The feed streams were mixed $1: 10$ against an excess of Milli-Q water and characterized for size and FL immediately afterward. The NCs were diluted in water for DLS measurements, and multiple dilutions were measured by FL to establish the linearity of FL versus [NC].

\subsubsection{Determination of NC size by dynamic light scattering}

Approximately $0.1 \mathrm{~mL}$ of ICG NC suspension was mixed with at least $2 \mathrm{~mL}$ of Milli-Q water to dilute the suspension to the point of water clarity in a low-volume PS cuvette (ZEN0112, Malvern Instruments, Boston). The cuvette was placed in a Malvern Zetasizer Nano ZS 3600 instrument (Malvern Instruments, Boston), and the intensity-weighted hydrodynamic size distribution ${ }^{63}$ of the NCs was determined by DLS. The Zetasizer analysis program in "normal mode" was used for the size determination.

\subsubsection{Zeta potential measurements}

NCs were formed with 10 wt. \% loading of ICG-C in $_{8}$ situ as described earlier in this section. The NCs were diluted 10 -fold into $1 \times$ PBS (Invitrogen) and loaded into capillary cells for zeta potential measurements on a Malvern Zetasizer ZEN3600 (Malvern Instruments, Massachusetts). The conductivity of the 
solution was $\sim 17.6 \pm 0.6 \mathrm{mS} / \mathrm{cm}$ and, the temperature was $25^{\circ} \mathrm{C}$.

\subsubsection{Fluorescence measurements}

NC suspensions were diluted with Milli-Q water by varying amounts such that the curve of FL versus NC concentration was linear. This was necessary to minimize the effects of NC light scattering on the FL measurements made. The FL per $\mathrm{NC}$ was calculated according to the procedure in Pansare et al. ${ }^{46}$ by dividing the FL value by the average number of NCs in suspension.

\subsubsection{ICG NC-BSA equilibration time course}

ICG- $\mathrm{C}_{4}, \mathrm{C}_{8}$, and $\mathrm{C}_{12}$ in situ complex and ICG-C $\mathrm{C}_{8}$ premade complex NCs were formed according to previously outlined methods at $10 \mathrm{wt}$. \% complex core loading and $20 \mathrm{mg} / \mathrm{mL}$ total solids in THF. The samples were dialyzed for $24 \mathrm{~h}$ to remove THF, and the size and ICG concentration were determined by DLS and UV-vis absorbance spectroscopy, respectively. $5 \mathrm{mM}$ phosphate was added to each sample, and the $\mathrm{pH}$ adjusted to $7.0 \pm 0.1$. BSA was then added to give $40 \mathrm{mg} / \mathrm{mL}$ in solution at room temperature, and FL time points were taken at $1001 \times$ dilution several times over $2 \mathrm{~h}$. The time course was kept to under $2 \mathrm{~h}$ for three reasons: (1) FL plateaus reached their maximum values and were stable in less than $30 \mathrm{~min}$, (2) ICG clears quickly from the body with a two-phase plasma half-life of 3 to $5 \mathrm{~min}$ in the initial phase and a 30 to $50 \mathrm{~min}$ in the secondary phase, ${ }^{63}$ and (3) it would be difficult to separate the effects of FL bleaching/degradation and ICG binding to BSA over a longer time course such as a 48-h study.

\subsubsection{BSA sink concentration variation}

Dialyzed ICG- $\mathrm{C}_{8}$ in situ complex nanoparticle suspensions with $5 \mathrm{mM}$ phosphate at $\mathrm{pH} 7.0$ were incubated with 40,60 , and $80 \mathrm{mg} / \mathrm{mL}$ BSA at room temperature, and FL time points were recorded over $2 \mathrm{~h}$.

\section{Disclosures}

The authors have no relevant financial interests in this paper and no potential conflicts of interest to disclose.

\section{Acknowledgments}

We acknowledge the financial support from the National Institutes of Health (Award No. 1RO1CA155061-1) and the Stuart M. Essig '83 and Erin S. Enright '82 Fund for Innovation in Engineering and Neuroscience.

\section{References}

1. V. J. Pansare et al., "Review of long-wavelength optical and NIR imaging materials: contrast agents, fluorophores, and multifunctional nano carriers," Chem. Mater. 24(5), 812-827 (2012).

2. S. M. Ansell et al., "Modulating the therapeutic activity of nanoparticle delivered paclitaxel by manipulating the hydrophobicity of prodrug conjugates," J. Med. Chem. 51(11), 3288-3296 (2008).

3. M. E. Davis, Z. Chen, and D. M. Shin, "Nanoparticle therapeutics: an emerging treatment modality for cancer," Nat. Rev. Drug Discovery 7(9), 771-782 (2008).

4. V. Kumar et al., "Stabilization of the nitric oxide (NO) prodrugs and anticancer leads, PABA/NO and double JS-K, through incorporation into PEG-protected nanoparticles," Mol. Pharm. 7(1), 291-298 (2009).
5. V. Kumar et al., "Formulation and stability of itraconazole and odanacatib nanoparticles: governing physical parameters," Mol. Pharm. 6(4), 1118-1124 (2009).

6. C. L. Amiot et al., "Near-infrared fluorescent materials for sensing of biological targets," Sensors 8(5), 3082-3105 (2008).

7. M. Gumbleton and D. J. Stephens, "Coming out of the dark: the evolving role of fluorescence imaging in drug delivery research," Adv. Drug Delivery Rev. 57(1), 5-15 (2005).

8. V. B. Rodriguez et al., "Encapsulation and stabilization of indocyanine green within poly (styrene-alt-maleic anhydride) block-poly (styrene) micelles for near-infrared imaging," J. Biomed. Opt. 13(1), 014025 (2008).

9. M. Akbulut et al., "Generic method of preparing multifunctional fluorescent nanoparticles using flash nanoprecipitation," Adv. Funct. Mater. 19(5), 718-725 (2009).

10. R. Bardhan et al., "Theranostic nanoshells: from probe design to imaging and treatment of cancer," Acc. Chem. Res. 44(10), 936-946 (2011).

11. W. Chen et al., "A molecularly targeted theranostic probe for ovarian cancer," Mol. Cancer Ther. 9(4), 1028-1038 (2010).

12. S. M. Janib, A. S. Moses, and J. A. MacKay, "Imaging and drug delivery using theranostic nanoparticles," Adv. Drug Delivery Rev. 62(11), 1052-1063 (2010).

13. C. Sanson et al., "Doxorubicin loaded magnetic polymersomes: theranostic nanocarriers for MR imaging and magneto-chemotherapy," ACS Nano 5(2), 1122-1140 (2011).

14. S. Santra et al., "Drug/dye-loaded, multifunctional iron oxide nanoparticles for combined targeted cancer therapy and dual optical/magnetic resonance imaging," Small 5(16), 1862-1868 (2009).

15. X. Zheng et al., "Indocyanine green-containing nanostructure as near infrared dual-functional targeting probes for optical imaging and photothermal therapy," Mol. Pharm. 8(2), 447-456 (2011).

16. J. Gunn et al., "A pretargeted nanoparticle system for tumor cell labeling," Mol. Biosyst. 7(3), 742-748 (2011).

17. J. Gunn et al., "A multimodal targeting nanoparticle for selectively labeling T cells," Small 4(6), 712-715 (2008).

18. P. A. Jarzyna et al., "Iron oxide core oil-in-water emulsions as a multifunctional nanoparticle platform for tumor targeting and imaging," Biomaterials 30(36), 6947-6954 (2009).

19. L. Jun et al., "Biodegradable nanoparticles for targeted ultrasound imaging of breast cancer cells in vitro," Phys. Med. Biol. 52(16), 47394747 (2007).

20. A. M. Koch et al., "Uptake and metabolism of a dual fluorochrome Tat-nanoparticle in HeLa cells," Bioconjugate Chem. 14(6), 1115-1121 (2003).

21. T. Parikh, M. M. Bommana, and E. Squillante, "Efficacy of surface charge in targeting pegylated nanoparticles of sulpiride to the brain," Eur. J. Pharm. Biopharm. 74(3), 442-450 (2010).

22. G. R. Reddy et al., "Vascular targeted nanoparticles for imaging and treatment of brain tumors," Clin. Cancer Res. 12(22), 6677-6686 (2006).

23. N. Zhang et al., "PLGA nanoparticle-peptide conjugate effectively targets intercellular cell-adhesion molecule-1," Bioconjugate Chem. 19(1), 145-152 (2008).

24. M. Landsman et al., "Light-absorbing properties, stability, and spectral stabilization of indocyanine green,” J. Appl. Physiol 40(4), 575-583 (1976).

25. S. T. Proulx et al., "Quantitative imaging of lymphatic function with liposomal indocyanine green," Cancer Res. 70(18), 7053-7062 (2010).

26. J.-M. Devoisselle et al., "Fluorescence properties of indocyanin green: I. In-vitro study with micelles and liposomes," Proc. SPIE 2980, 453-460 (1997).

27. T. H. Kim et al., "Evaluation of temperature-sensitive, indocyanine green-encapsulating micelles for noninvasive near-infrared tumor imaging," Pharm. Res. 27(9), 1900-1913 (2010).

28. A.-K. Kirchherr, A. Briel, and K. Mäder, "Stabilization of indocyanine green by encapsulation within micellar systems," Mol. Pharm. 6(2), 480-491 (2009).

29. V. B. Rodriguez et al., "Encapsulation and stabilization of indocyanine green within poly(styrene-alt-maleic anhydride) block-poly(styrene) micelles for near-infrared imaging," J. Biomed. Opt. 13(1), 014025 (2008).

30. G. Sun et al., "Bright fluorescent nanoparticles for developing potential optical imaging contrast agents," Nanoscale 2(4), 548 (2010). 
31. S. Gupta et al., "Effect of nano-encapsulation on photophysical properties of ICG," Proc. SPIE 7910, 79100U (2011).

32. J. Yu et al., "Self-assembly synthesis, tumor cell targeting, and photothermal capabilities of antibody-coated indocyanine green nanocapsules," J. Am. Chem. Soc. 132(6), 1929-1938 (2010).

33. M. A. Yaseen et al., "Stability assessment of indocyanine green within dextran-coated mesocapsules by absorbance spectroscopy," J. Biomed. Opt. 12(6), 064031 (2007).

34. F. Tam et al., "Plasmonic enhancement of molecular fluorescence," Nano Lett. 7(2), 496-501 (2007).

35. R. Bardhan et al., "Tracking of multimodal therapeutic nanocomplexes targeting breast cancer in vivo," Nano Lett. 10(12), 4920-4928 (2010).

36. V. Saxena, M. Sadoqi, and J. Shao, "Indocyanine green-loaded biodegradable nanoparticles: preparation, physicochemical characterization and in vitro release," Int. J. Pharm. 278(2), 293-301 (2004).

37. V. Saxena, M. Sadoqi, and J. Shao, "Enhanced photo-stability, thermalstability and aqueous-stability of indocyanine green in polymeric nanoparticulate systems," J. Photochem. Photobiol., B 74(1), 29-38 (2004).

38. C. H. Lee et al., "Near-infrared mesoporous silica nanoparticles for optical imaging: characterization and in vivo biodistribution," Adv. Funct. Mater. 19(2), 215-222 (2009).

39. R. Benson and H. Kues, "Fluorescence properties of indocyanine green as related to angiography," Phys. Med. Biol. 23(1), 159-163 (1978).

40. Y. Uchiyama et al., "Role of the liquid/liquid interface in a phase-transfer catalytic reaction as investigated by in situ measurements using the quasi-elastic laser scattering method," Langmuir 16(16), 6597-6600 (2000).

41. R. Philip et al., "Absorption and fluorescence spectroscopic investigation of indocyanine green," J. Photochem. Photobiol. A 96(1), 137-148 (1996).

42. H. K. Yoon et al., "Polymer-protein hydrogel nanomatrix for stabilization of indocyanine green towards targeted fluorescence and photoacoustic bio-imaging," J. Mater. Chem. B 1(41), 5611-5619 (2013).

43. B. K. Johnson and R. K. Prud'homme, "Flash nanoprecipitation of organic actives and block copolymers using a confined impinging jets mixer," Aust. J. Chem. 56(10), 1021-1024 (2003).

44. S. M. D'Addio et al., "Effects of block copolymer properties on nanocarrier protection from in vivo clearance," J. Controlled Release 162(1), 208-217 (2012).

45. Y. Liu, Z. Tong, and R. K. Prud'homme, "Stabilized polymeric nanoparticles for controlled and efficient release of bifenthrin," Pest Manage. Sci. 64(8), 808-812 (2008).

46. V. J. Pansare et al., "Composite fluorescent nanoparticles for biomedical imaging," Mol. Imaging Biol. 16(2), 180-188 (2014).

47. S. J. Budijono et al., "Synthesis of stable block-copolymer-protected NaYF4:Yb3+, Er3+ Up-converting phosphor nanoparticles," Chem. Mater. 22(2), 311-318 (2009).

48. N. M. Pinkerton et al., "Formation of stable nanocarriers by in situ ion pairing during block-copolymer-directed rapid precipitation," Mol. Pharm. 10(1), 319-328 (2012).

49. J. F. Zhou, M. P. Chin, and S. A. Schafer, "Aggregation and degradation of indocyanine green," Proc. SPIE 2128, 495-505 (1994).

50. T. Förster, "Excimers," Angew. Chem., Int. Ed. Engl. 8(5), 333-343 (1969).

51. V. Kumar, D. H. Adamson, and R. K. Prud'homme, "Fluorescent polymeric nanoparticles: aggregation and phase behavior of pyrene and amphotericin B molecules in nanoparticle cores," Small 6(24), 29072914 (2010).

52. B. K. Johnson and R. K. Prud'homme, "Mechanism for rapid selfassembly of block copolymer nanoparticles," Phys. Rev. Lett. 91(11), 118302 (2003).

53. A. D. 1. Zerda et al., "Ultrahigh sensitivity carbon nanotube agents for photoacoustic molecular imaging in living mice," Nano Lett. 10(6), 2168-2172 (2010).
54. S. Zanganeh et al., "Photoacoustic imaging enhanced by indocyanine green-conjugated single-wall carbon nanotubes," J. Biomed. Opt. 18(9), 096006 (2013).

55. ChemAxon, "MarvinSketch geometry plugin," version 6.2.2, 2014, http://www.chemaxon.com/products/marvin/marvinsketch.

56. G. R. Cherrick et al., "Indocyanine green: observations on its physical properties, plasma decay, and hepatic extraction," J. Clin. Invest. 39(4), 592-600 (1960).

57. S. Mordon et al., "Indocyanine green: physicochemical factors affecting its fluorescence in vivo," Microvasc. Res. 55(2), 146-152 (1998).

58. T. J. Muckle, "Plasma proteins binding of indocyanine green," Biochem. Med. 15(1), 17-21 (1976).

59. S. Yoneya et al., "Binding properties of indocyanine green in human blood," Invest. Ophthalmol. Visual Sci. 39(7), 1286-1290 (1998).

60. F. Rotermund, R. Weigand, and A. Penzkofer, "J-aggregation and disaggregation of indocyanine green in water," Chem. Phys. 220(3), 385-392 (1997).

61. R. J. Porter and R. B. Layzer, "Plasma albumin concentration and diphenylhydantoin binding in man," Arch. Neurol. 32(5), 298-303 (1975).

62. D. Adamson, "Convenient method for the preparation of poly (ethylene oxide) and poly (ethylene oxide) block copolymers," Polym. Prepr. 41(2), 1231-1232 (2000).

63. T. Desmettre, J. M. Devoisselle, and S. Mordon, "Fluorescence properties and metabolic features of indocyanine green (ICG) as related to angiography," Surv. Ophthalmol., 45(1), 15-27 (2000).

Vikram J. Pansare is a recent $\mathrm{PhD}$ graduate in the Department of Chemical and Biological Engineering at Princeton University. During his $\mathrm{PhD}$, he published papers in biomedical imaging, fundamental colloidal phenomena, and nanoparticle processing at industrial scales. Following a 2-year position as a materials startup founder and CTO, he now works at McKinsey \& Co. serving clients across a range of scientific industries.

William J. Faenza is the CEO/CTO of Persis Science LLC-based in Princeton, New Jersey. As an entrepreneur for many years, his inventions and scientific work have impacted many Fortune 500 companies and academic collaborations. A chemist by training, his research interests include security printing, biomedical imaging, and total synthesis of chiral molecules.

Hoang Lu received his BS degree in chemical engineering from Columbia University, where he conducted protein engineering research in Professor Scott Banta's Group, and his MSE in bioengineering from the University of Pennsylvania, where he worked on biomaterials research in Professor Jason Burdick's lab. He is a $\mathrm{PhD}$ candidate in the Lab of Prof. R. K. Prud'homme at Princeton University. He has a minor in East Asian studies and has also worked on DNA nanotechnology research in Professor Peng Yin's Lab at Harvard University. The focus of his current research is on engineering nanoparticles for targeted drug delivery and diagnostics.

Douglas H. Adamson is an associate professor at the Institute of Materials Science, University of Connecticut. His research interests are focused on bioinspired materials, polymersomes, and nanofillers. He has authored more than 190 publications and received more than 11,000 citations.

Robert K. Prud'homme is a professor of chemical and biological engineering at Princeton University. His research interests span many fields from complex fluids, polymer-wax interactions, and colloidal phenomena to nanoparticle drug delivery and biomedical imaging. $\mathrm{He}$ is an author of more than 250 peer-reviewed publications and an inventor with more than 20 patents. 